\title{
Graphical thresholding procedure and optimal light level estimation for spatially resolved photon counting with EMCCDs
}

\author{
Ottavia Jedrkiewicz $^{\text {a,* }}$, Jean-Luc Blanchet ${ }^{\text {a }}$, Eric Lantz ${ }^{\text {b }}$, Paolo Di Trapani ${ }^{\text {a }}$ \\ a CNISM and Dipartimento di Fisica e Matematica, Università dell'Insubria, Via Valleggio 11, 22100 Como, Italy \\ ${ }^{\mathrm{b}}$ Institut FEMTO-ST, Laboratoire d'Optique P.M. Duffieux, UMR CNRS 6174, Université de Franche-Comté, 16 route de Gray, F-25030, Besançon Cedex, France
}

\section{A R T I C L E I N F O}

Article history:

Received 25 January 2011

Received in revised form 21 July 2011

Accepted 15 September 2011

Available online 1 October 2011

\section{Keywords:}

EMCCD detection

Photon counting

Quantum optics

\begin{abstract}
A B S T R A C T
Thanks to the electron multiplying function that can effectively convert the weak incident photon signal to amplified electron output, electron multiplying charged-coupled devices (EMCCDs) are becoming useful and popular detectors in photon counting regimes necessitating also spatial resolution. A multi-imaging strategy has been already proposed and experimentally tested to improve the accuracy of photon counting with an EMCCD, by taking into account the random nature of its on-chip gain and the possibility of multiple photodetection events on 1 pixel. In this paper, referring to the thresholding procedure developed for photon counting, we present a clear graphical method for the threshold and the optimal light level estimations. Thanks to the graphical visualization of the probabilities involved in the detection errors on 1 pixel, we are able to derive in a straightforward way and for any EMCCD, the threshold level and thus the best mean level of illumination to be used in order to minimize the false detection probabilities that might ruin the image statistics, especially in cases where quantum spatial effects might be observed.
\end{abstract}

(c) 2011 Elsevier B.V. All rights reserved.

\section{Introduction}

Electron multiplying charge-coupled devices (EMCCDs) use impact ionization to provide high gain in the charge domain, i.e. they apply a gain to the pixel's charge before it reaches the output amplifier [1]. This enables imaging with an equivalent input noise of much less than $1 \mathrm{rms}$ electron at pixel rates up to and beyond those required for TV imaging applications. The ultra-low noise, high resolution, high quantum efficiency and robustness to overexposure make these sensors ideally suited to applications traditionally served by image intensifiers. In fact since EMCCDs were first produced in the late 1990s, they have found use in many applications where high frame rate imaging is required. These include surveillance, LIDAR applications, astronomy, and other scientific imaging applications such as for instance very low level bioluminescence for drug discovery and genetic engineering applications. Recently, EMCCDs have also been used to record radiation patterns generated from optical parametric down conversion processes in low gain regime, and to analyze the performances of these cameras for the detection of single photons from the study of the spatial correlation of the entangled twin beams [2-4]. Indeed, in EMCCDs, amplification occurs before reading in a multiplication register that contains several hundred of cells. Electrons are shifted from one cell to another with a small probability in each cell of being duplicated, resulting in a high mean gain typically of the order of 1000 . Because of this high gain,

\footnotetext{
* Corresponding author. Tel.: + 39 0312386257; fax: + 390312386209. E-mail address: Ottavia.Jedrkiewicz@uninsubria.it (O. Jedrkiewicz).
}

even the signal generated from a single photon emerges from the readout noise floor with high probability. However, although the effective gain is very large, the detailed process by which the signal is amplified is stochastic and introduces therefore additional noise at the output. In other words it is not possible to assign a well defined number of photons to each value of the output signal. The basic principle of charge multiplication in the EMCCD was studied several times theoretically (see for instance $[5,6]$ ), and it was shown also experimentally [7] that the division of the output signal by the mean gain results in adding a Poisson detection noise, called excess noise, having the same amplitude as the photon noise. Basden et al. [8] have proposed a photon counting strategy to partially remove this noise for low light level images, in the ideal case where the noise comes only from the random gain (without considering the readout noise and the clock-induced-charge noise), by using a "photometric correction" that uses a mean level of light. More recently a multi-imaging strategy has been proposed and experimentally tested to improve the accuracy of photon counting with an EMCCD, by taking into account the random nature of its on-chip gain and the possibility of multiple photodetection events on 1 pixel [9]. That strategy is based on Bayesian estimation on each image, with a priori information given by the sum of the images. The work presented in Ref. [9] is based on the development of a thresholding procedure for photon counting with EMCCDs. In the present manuscript, referring to that procedure, which we recall in the next section, we present a clear graphical method for the threshold and optimal light level estimations. Thanks to the graphical visualization of the probabilities involved in the detection errors on 1 pixel, we are able to derive in a straightforward way and 
for any EMCCD, the threshold level and thus the best mean level of illumination to be used in order to minimize the false detection probabilities that might ruin the real image statistics.

\section{Thresholding and light level evaluation: a brief review}

In general, given $n$ photoelectrons (pe-) at its input, the multiplication register of the EMCCD provides a random output of $x$ electrons, with a distribution approximately described by [8]:

$p(x \mid n)=\frac{x^{n-1} e^{-x / g}}{g^{n}(n-1) !}$,

when the photon input level is relatively small and the mean gain $g$ is large. For simplicity, in the following we assume that the quantum efficiency of the detector is unity, and thus we can speak about input photons instead of photoelectrons. Because of the stochastic process of amplification, it is not possible to assign a precise input value $n$ on each pixel given the recorded value of $x$, as it also appears from the representation of the conditional probabilities of $x$ given $n$, shown in Fig. 1. In other words there is always some uncertainty when estimating the input photon number. Note that only in the ideal case of (i) absence of noise and (ii) very low mean level of illumination, leading to at most one photon per pixel, the presence of an output signal (of random height) can be taken as the certain indication of the presence of one input photon. The second ideal case where (i) noise is still absent but (ii) the level of illumination of the image to be detected is arbitrary large can be straightforwardly reduced to the previous one by decreasing the acquisition time $\Delta t$ to a sufficiently small value to guarantee the "at most one photon per pixel condition" to be fulfilled. In this circumstance, by recording a large number $N$ of statistically identical shots and by subsequent adding the number of counts per pixel, the image (i.e. the average spatial distribution of photon numbers) is exactly retrieved without incurring in the errors that would appear for longer acquisition times due to multiple photons on one pixel in the same shot. In the absence of noise, image detection can be made exact in the limit of extremely low level of illumination per shot.

The reality is of course different because of the presence of different noises, like the readout noise and the clock-induced-charge (CIC) noise, leading in this case to an output different from zero in the absence of photons. In fact the relative contribution of the noise increases on

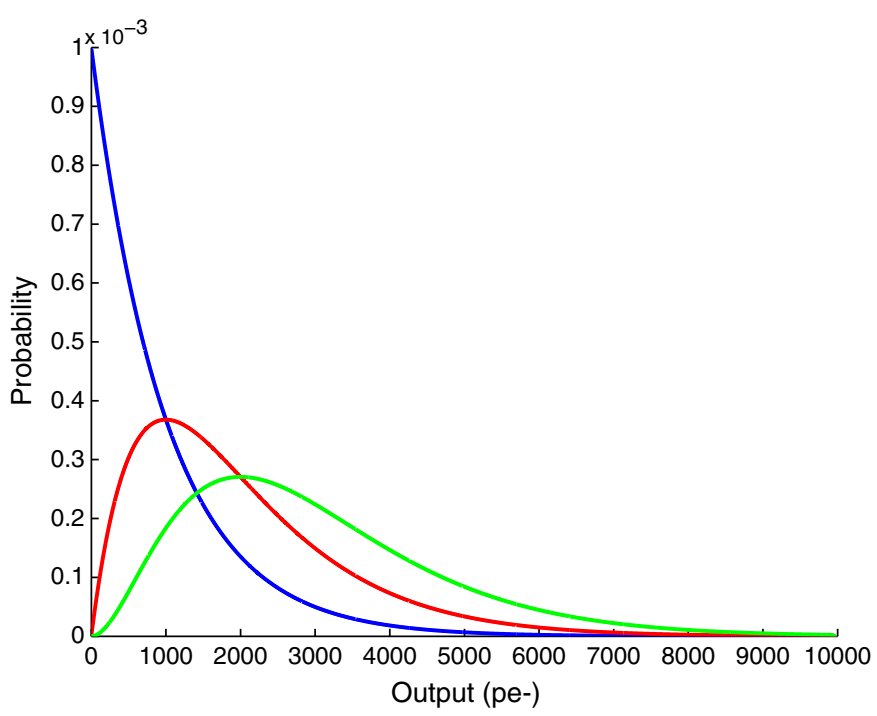

Fig. 1. EMCCD output probabilities for a given number of input photons $n=1$ (blue curve), $n=2$ (red curve), and $n=3$ (green curve), and for a mean gain of 1000 . decreasing the level of illumination per shot. This reasoning indicates the existence of an optimum level of illumination for which the total error due to both multiple-photon and noise-induced fake detections is minimal. Notably, besides the knowledge about this optimum illumination what is necessary is a procedure for defining the output-signal threshold below which the event has to be taken as caused by the noise and above which as caused by one photon. In Ref. [9] both the optimum illumination level and the threshold are obtained from a single mathematical procedure by minimizing the error caused by all fake detections in the two-parameter space, as outlined here below. Note that the procedure holds for each independent pixel, since different levels of average illumination due to image in-homogeneity lead to different results. The thresholding procedure developed by Lantz and coworkers [9] consists in the following: on each image, assumed to have a mean light level per pixel $<<1$, they determine a value $T$ of the threshold and decide there is an input photon if the output in grey levels (" $g l ", 1 g l=12$ electrons for the Andor camera at maximum gain: see below) is greater than $T$. Therefore if $x_{g l}>T, y=1$ is the result of the thresholding procedure. Alternatively if $x_{g l}<T$, there is no input photon and the result of the procedure gives $y=0$. Here are summarized the false detections events leading to errors (in the following $x$ designates a number of grey levels): 1) $x>T$ for $n=0$ (due to noise): one photon is detected instead of 0 , with probability $p(y=1 \mid 0) \equiv p_{10}$.

2) $x<T$ for $n=1$ (due to random gain): 0 photons are detected instead of 1 , with probability $p(y=0 \mid 1) \equiv p_{01}$.

3) $x>T$ for $\mathrm{n}=2: 1$ photon is detected instead of 2 , with probability $p(y=1 \mid 2) \equiv p_{12}$. The case $x<T$ for $\mathrm{n}=2$ can be considered negligible. In general, from the choice of the number of images $N$ that can be recorded, we can find the approximate light level $\mu=<n>/ N$ needed on each image for a mean photon number $\langle n>$ on the sum. The procedure to derive these quantities is the minimization of the quadratic error $Q$ for one pixel on the sum of the images, as done in Ref. [9]. By using this criterion, equal weighting is assumed for false positive and false negative events. It would be straightforward to generalize to an experiment where physical considerations lead to different weighting for these events: the above probabilities would be simply multiplied by their respective weighting and all the subsequent derivation would be identical. Since for low $\mu$ the statistics of the incoming light may be considered as Poissonian, $Q$ can be written as:

$$
\begin{aligned}
Q & =N\left[p_{01} \mu+p_{10}(1-\mu)+p_{12} \frac{\mu^{2}}{2}\right] \\
& =<n>p_{01}+(N-<n>) p_{10}+\frac{<n>}{2 N} p_{12},
\end{aligned}
$$

where $p_{01}, p_{10}$ and $p_{12}$ are precisely the repartition functions of the conditional probabilities of errors, thus respectively defined as:

$$
\begin{aligned}
& p_{01}=\sum_{x=0}^{T} p(x \mid 1), \\
& p_{10}=\sum_{x>T}^{\infty} p(x \mid 0), \\
& p_{12}=\sum_{x>T}^{\infty} p(x \mid 2) .
\end{aligned}
$$

Note that in Eqs. (3) and (5) the conditional probabilities are given by the distribution (Eq. 1), while in Eq. (4) $p(x \mid 0)$ is an experimental probability distribution that can be derived from the histogram of grey levels obtained from a dark image of the EMCCD with shutter closed. This histogram gives information on the noises involved in the detection, since it can be fitted by a theoretical probability law by using a Gauss-Newton algorithm which highlights the readout 
noise contribution from the Gaussian fit and the two CIC contributions of the serial and parallel register respectively, from the long following tail as shown in Ref. [9]. In that work the grey level histogram, which is shown for illustration in Fig. 2, was obtained by using an EMCCD camera from Andor Technologies (IXON, sensor model E2V CCD97, Head 897E CS0 n.BV), operated with an exposure time of $33 \mathrm{~ms}$, cooled down to $-85^{\circ} \mathrm{C}$, and with a pixel readout rate of $10 \mathrm{MHz}$ and a vertical clock speed of one shift $/ 0.5 \mu$ s. The dynamic range of this EMCCD was 14 bit and the gain $g$ was 1000 in photoelectrons or $1000 / 12$ in grey levels $(1 g l=12 p e)$. It is worth mentioning that the camera used was selected by Andor, under special request, among a series of EMCCDs produced, with the characteristics of having CIC values ( pix $^{-1}$ frame ${ }^{-1}$ ) much lower than the average standard ones. Finally note that the spatial statistics of a dark image gives for that camera a mean number of grey levels of 600 with a total experimental standard deviation of $9.7 \mathrm{gl}$ (corresponding to $9.7 \times 12 / 1000=0.12 \mathrm{ph} / \mathrm{pix}$ ).

The procedure of minimization of $Q$ is performed with respect to the variables $N$ and $T$. So considering $T / g<<1$ and thus from Eq. (3) (when approximated by an integral)

$p_{01}=1-e^{-T / g} \cong T / g$,

and using also the approximations $N-<n>\approx N$ and $p_{12} \cong 1$, the minimization leads to the following results [9]:

$\mu=\frac{<n>}{N}=\sqrt{2 p_{10}}$,

and $T$ is such that

$p_{10}=\frac{g^{2}}{2}\left(\frac{\partial p_{10}}{\partial T}\right)^{2}$

While the value of $\mu$ from Eq. (7) can be obtained for a given $T$ through the evaluation of the sum in Eq. (4) with the use of the experimental data, the threshold $T$ is less straightforward to calculate because of the absence of formula for $p_{10}$. To find $T$, the derivative in Eq. (8) is evaluated in a lengthy way, by calculating $p_{10}$ for each value of $T$ so to calculate progressively the finite differences $\left(p_{10}(T+1)-p_{10}\right.$ $(T-1)) / 2$. We conclude by summarizing the results obtained from this analytical thresholding procedure, since we shall compare them with the results that will be presented in the next section. For the

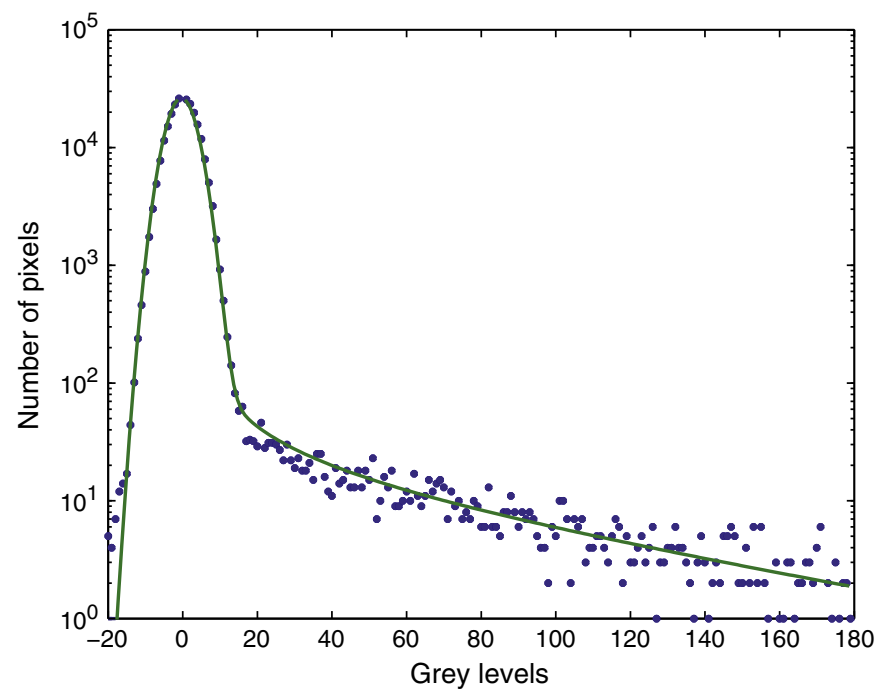

Fig. 2. Histogram of grey levels for a dark image of the Andor EMCCD (blue dots) and corresponding fit (green curve).
Andor EMCCD under consideration, Lantz and coworkers [9] estimated that in order to correctly work in single photon counting regime and to minimize the error probabilities during detection, the mean number of photons per pixel (per image) needed is $\mu=0.15 \mathrm{ph} / \mathrm{pix}$ and the threshold value is $T=11 \mathrm{gl}$. Each of the three errors has in this case probabilities respectively of the order of 1 or $2 \%$ at most.

\section{Graphical method for the thresholding procedure}

The graphical method proposed here is based on the simultaneous visualization of the three probability functions that are directly involved in the detection errors on 1 pixel. Assuming a low mean illumination level on the single image, we consider as before $\mu<<1$ and a Poissonian distribution of input light. The above mentioned probabilities in the single photon detection regime of an EMCCD are thus $p(x \mid 0)(1-\mu), p(x \mid 1) \mu$, and $p(x \mid 2) \mu^{2} / 2$. By superposing the corresponding curves plotted as a function of the output grey levels $x$, we shall see how the threshold value $T$ to be used in the detection procedure can be extracted from the analysis of their respective crossings.

\subsection{Estimation of $T$ for a given illumination level}

The probability of recording $x$ grey levels when there are no input photons is $p(x \mid 0)$, which can be derived as mentioned earlier from the histogram of grey levels obtained from a dark image of the EMCCD. To be sure that we represent a probability that will have to be compared with the probabilities of recording $x$ when there are 1 or 2 photons per pixel in the input, the histogram shown in Fig. 2 should be renormalized, by dividing the values by the total number of pixels contained in the camera sensor. In Fig. 3 we have plotted on the same scale the three curves describing the probabilities of recording $x$, for 0,1 or 2 photons per pixel, in three different cases of illumination, for the Andor camera described before. Note that the histogram has been replaced by a continuous curve to facilitate the visualization of its trend. On the other hand, in order to represent all the probabilities versus grey levels, the functions $p(x \mid 1)$, and $p(x \mid 2)$ are obtained from Eq. (1) with the gain now expressed as $g=1000 / 12$, having taken into account the conversion factor of the camera. The mean number $\mu$ of input photons per pixel per image is treated as a parameter.

By analyzing the plots presented in Fig. 3, we can observe that the probabilities $p(x \mid 0)(1-\mu)$ and $p(x \mid 1) \mu$ associated respectively with the detection of $x$ grey levels for 0 and 1 input photons, have an intersection point at $x_{\text {int } 1}$ which, for decreasing input mean photon numbers, is characterized by increasing values of grey levels. In the limit of $\mu \rightarrow 0$, this intersection point moves towards right "inside" the CIC region of the experimental dark image curve. On the other hand we note that the curve describing $p(x \mid 2) \mu^{2} / 2$ associated with the probability of detecting $x$ grey levels given 2 photons per pixel generally remains below the curve $p(x \mid 1) \mu$, but crosses the probability $p(x \mid 0)$ $(1-\mu)$ for a value of grey levels $x=x_{\text {int } 2}>x_{\text {int } 1}$.

The search for a criterion of choice for the threshold $T$ should start from its definition, that is that $T$ must be such that if $x>T$ we decide that there is one input photon, otherwise there is no input photon. In fact, from the observations made above we can intuitively say that at the intersection point defined by $x_{\text {int } 1}$, we should be able to distinguish between dark noise and a real photon arriving. On the other hand, if the input illumination level is too low with $\mu \rightarrow 0$, light levels of the order of the CIC $\left(5.10^{-3}\right.$ pix $^{-1}$ frame $^{-1}$ for the particular Andor EMCCD under consideration) cannot be distinguished from the CIC noise. Typically one can see that the distinction starts to become difficult at $\mu=10^{-2} \mathrm{ph} / \mathrm{pix}$. Thus $\mu$, which for the moment is being treated as a parameter, should lie in any case above the CIC noise level. Given these considerations, it turns out that for a certain value of $\mu, T=x_{\text {int } 1}$ is the correct choice. On the other hand we must ensure that the probability of detecting 2 photons per pixel 

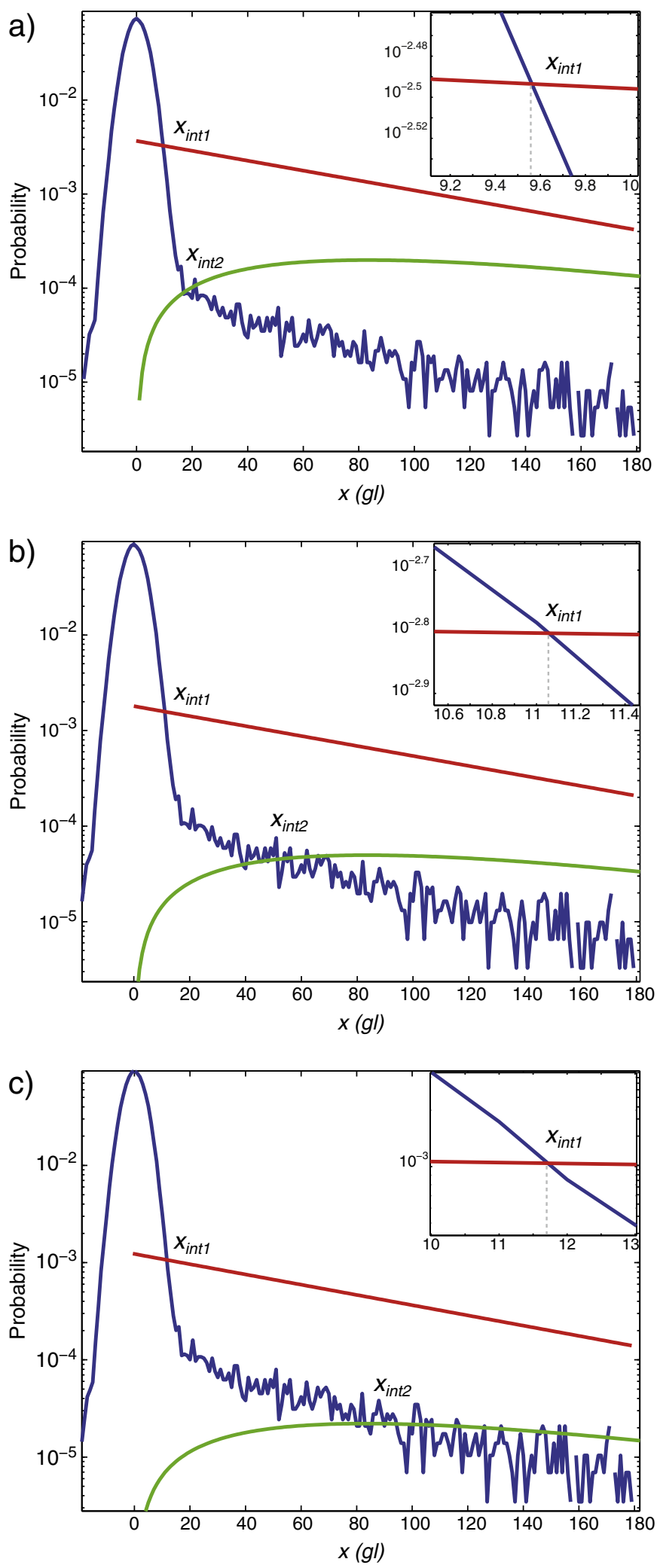

Fig. 3. Probabilities $\left(p(x \mid 0)(1-\mu)\right.$ in blue, $p(x \mid 1) \mu$ in red, and $p(x \mid 2) \mu^{2} / 2$ in green) involved in the thresholding procedure for the Andor EMCCD, for three different cases of illumination; (a) $\mu=0.3 \mathrm{ph} / \mathrm{pix}$, (b) $\mu=0.15 \mathrm{ph} / \mathrm{pix}$, (c) $\mu=0.1 \mathrm{ph} / \mathrm{pix}$. In the inset, zoom of the region of intersection between $p(x \mid 0)(1-\mu)$ and $p(x \mid 1) \mu$. remains as low as possible, and thus we must have $\mu<<x_{\text {int2 }}$. The detailed procedure for the graphical thresholding just shown, together with the estimation of the optimal light level, will be summarized in the next section. For the moment, just note here how the value $x_{1 \text { cross }} \cong 11 \mathrm{gl}$ for a mean number of photons $\mu=0.15 \mathrm{ph} /$ pix (see Fig. $3 \mathrm{~b}$ ) is in agreement with the threshold value $T$ derived analytically in Ref. [9] in the same illumination conditions, which were found to minimize the quadratic error $Q$.

In the following, we show that for a given mean value of photons per pixel $\mu<<1$, the graphical method for the determination of $T$ here presented is equivalent to the thresholding procedure described in Ref. [9]. By combining Eqs. (7) and (8), we have the following relation:

$\sqrt{2 p_{10}}=\mu=g\left(-\frac{\partial p_{10}}{\partial T}\right)$.

On the other hand because of Eq. (6), $g=1 /\left(\partial p_{01} / \partial T\right)$, and thus

$\mu=\frac{-\partial p_{10} / \partial T}{\partial p_{01} / \partial T}$

Using the fact that $\partial p_{10} / \partial T=-p(x \mid 0)$ and $\partial p_{01} / \partial T=p(x \mid 1)$, we have from Eq. (10):

$\mu \frac{\partial p_{01}}{\partial T}=\mu p(x \mid 1)=\frac{-\partial p_{10}}{\partial T}=p(x \mid 0) \cong(1-\mu) p(x \mid 0)$

$\Rightarrow \mu p(x \mid 1)=(1-\mu) p(x \mid 0)$.

The equality given by Eq. (12) is equivalent to the condition of intersection of the functions $p(x \mid 1) \mu$ and $p(x \mid 0)(1-\mu)$, illustrated in the graphs of Fig. 3. In other words, for a given value of $\mu<<1, T$ is such that Eq. (12) is satisfied. Note that the graphical method as well as Eq. (12) gives a non integer value for the best threshold, while the number of grey levels is an integer. This non integer value $\mathrm{T}$ can be used without any difficulties by deciding no detection for $x_{g l}<T$ and detection for $x_{g l}>T$, where $x_{g l}$ is the integer number of grey levels.

3.2. Guidelines for the determination of $T$ and for the optimization of the mean light level

The aim of this work has been to present a straightforward graphical thresholding method to be applied when using the EMCDD in single photon detection regime. Let thus summarize the steps of the procedure:

i) At first, the mean illumination level of the single image to be recorded could be chosen in relation to the value of the EMCCD dark noise image standard deviation $\sigma_{\text {noise }}$, expressed in photons per pixel. This avoids to fall into the CIC noise level, which as mentioned before should be the lowest limit value. We can call $\mu_{\text {approx }}$ the value of $\mu$ chosen initially.

ii) The second step is the plot of the probability curves associated with the detection of $x$ grey levels for 0 , for 1 or for 2 photons per pixel, and the straightforward derivation of the threshold $T$ from the intersection point of the functions $p(x \mid 1) \mu$ and $p(x \mid 0)$ $(1-\mu)$, as shown before.

iii) The final step is the optimization of the light level $\mu$ from a comparison with the value obtained analytically from Eq. (7). This can be derived after evaluation of the repartition function from the experimental dark noise histogram, $p_{10}=\sum_{x>T}^{\infty} p(x \mid 0)$, where $T$ has the value estimated from step 2 . Indeed we can calculate $\mu_{1}=\sqrt{2 p_{10}}$ and compare it with the initially chosen value $\mu_{\text {approx. }}$. If $\mu_{1} \neq \mu_{\text {approx }}$ the graphical procedure to determine $T$ should be repeated by iteration with the new choice for the illumination level given by $\mu_{1}$. 
The optimal values for the threshold $T$ and for the illumination level $\mu_{\text {opt }}$ are clearly interlinked. These are such that for the optimum threshold, the mean number of photons per pixel $\mu$ is in good agreement with the calculated value from Eq. (7). It is very interesting to note that this iteration procedure reveals that the optimal level $\mu_{\text {opt }}$ is such that $\mu \geq \sigma_{\text {noise }} p h /$ pix and more precisely $\mu$ should be just above $\sigma_{\text {noise }}$. For the Andor camera under consideration we find with our method $\mu_{\text {opt }} \cong 0.13 \mathrm{ph} /$ pix with $T \cong 11 \mathrm{gl}$. Note that once optimized, the thresholding helps one estimate other input illumination levels if they are close to the initial estimated value.

\subsection{False detection probabilities}

In the following we present a graphical evaluation of the probabilities of errors in detection after the assessment of the optimal thresholding level $T$. By plotting the three false detection probabilities of interest, being respectively defined as

$P_{0} \equiv \sum_{x>T}^{\infty} p(x \mid 0) \times(1-\mu)$

when we record $x>T$ in the case where there are no input photons,

$P_{1} \equiv \sum_{x>0}^{T} p(x \mid 1) \times \mu$

when we record $x<T$ for 1 input photon, and

$P_{2} \equiv \sum_{x>T}^{\infty} p(x \mid 2) \times \frac{\mu^{2}}{2}$

when we record $x>T$ for 2 input photons (the case $x<T$ being negligible), we can also visualize and establish a reasonable range of $\mu$ to work with.

Fig. 4 shows the three false detection probabilities, together with the total probability of error on the pixel, plotted as a function of the mean number of photons per pixel $\mu$, and for a fixed value of the threshold $T$ (the graphs refer to the Andor EMCCD under consideration with $T=11$ ). The relative total error in detection (i.e. the total probability of error divided by $\mu$ ) is also shown (black curve). This relative error gives an estimation of the ratio of total false detections with respect to the true number of input photons, and it is a quantity that admits a minimum.

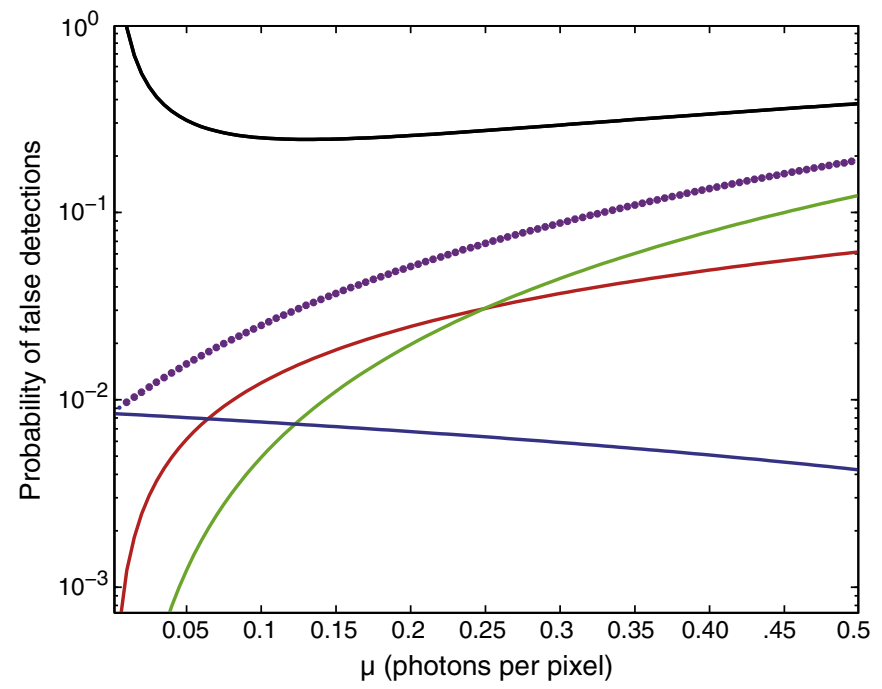

Fig. 4. False detection probabilities $P_{0}$ (blue curve), $P_{1}$ (red curve) and $P_{2}$ (green curve), and total probability of error (dotted curve), plotted as a function of $\mu$, for $T=11 \mathrm{gl}$ in the case of the Andor EMCCD. The black solid curve represents the relative total error in detection (i.e. total probability of error divided by $\mu$ ).
We start by noting that the relative total error reaches a minimum of about $24 \%$ and lies around this point for a given range of values of the mean number of input photons $\mu$. The minimum is associated with the value $\mu \cong 0.13 \mathrm{ph} / \mathrm{pix}$ (in accordance with the result of the graphical method), and for which the total probability of false detections is only about $3 \%$. In general acceptable values for the mean illumination level on a single image can be defined for instance as those that lead to a relative error below 0.25 . Also note that an alternative criterion for the definition of the range of acceptable values for $\mu$ can be that this range should have the lower and the upper limits defined respectively from the intersection of $P_{0}$ with $P_{1}$ and of $P_{1}$ with $P_{2}$. On one hand, we expect in fact that $P_{0}$ should stay below $P_{1}$, since for smaller values of $\mu$ where the probability of error $P_{0}$ is greater than $P_{1}$, the EMCCD noise becomes too large and the input photon cannot be detected. On the other hand, $\mu$ should be such that $P_{2}$ stays below $P_{1}$ otherwise the error in detection due to the arrival of 2 photons starts to be too high. From these considerations we can estimate from Fig. 4 that a good working region is given by values of $\mu$ in the range $0.06<\mu<0.25 \mathrm{ph} / \mathrm{pix}$.

\section{Application of the graphical thresholding method to a different EMCCD}

Until now we have described the graphical thresholding procedure applied to the same EMCCD used in the work of Lantz and coworkers [9], also in order to make a comparison with the results obtained in that work, and to highlight the rapidity and simplicity of the method here proposed.

In this section we illustrate the same graphical method applied to an EMCCD from Hamamatsu (Model C-9100). For that camera, which was a demonstration EMCCD, the mean gain $g$ was 1200 and the conversion factor $1 g l=5.8 p e$. The EMCCD was operated in similar conditions to those of the previous camera. The sensor (identical to the Andor one) was cooled down to $-80^{\circ} \mathrm{C}$, and the exposure time was $33 \mathrm{~ms}$ with a pixel readout rate of $11 \mathrm{MHz}$. This camera was characterized by a dynamic range of 16 bit. The dark image histogram of grey levels recorded with shutter closed is shown in Fig. 5. Note by comparing with Fig. 2 how the readout noise contribution is almost twice with respect to the case of the Andor. In general from the spatial statistics of the dark image, we have measured a standard deviation of the noise level of $39.5 \mathrm{gl}$, thus corresponding here to $39.5 \times 5.8 / 1200=0.19 \mathrm{ph} / \mathrm{pix}$.

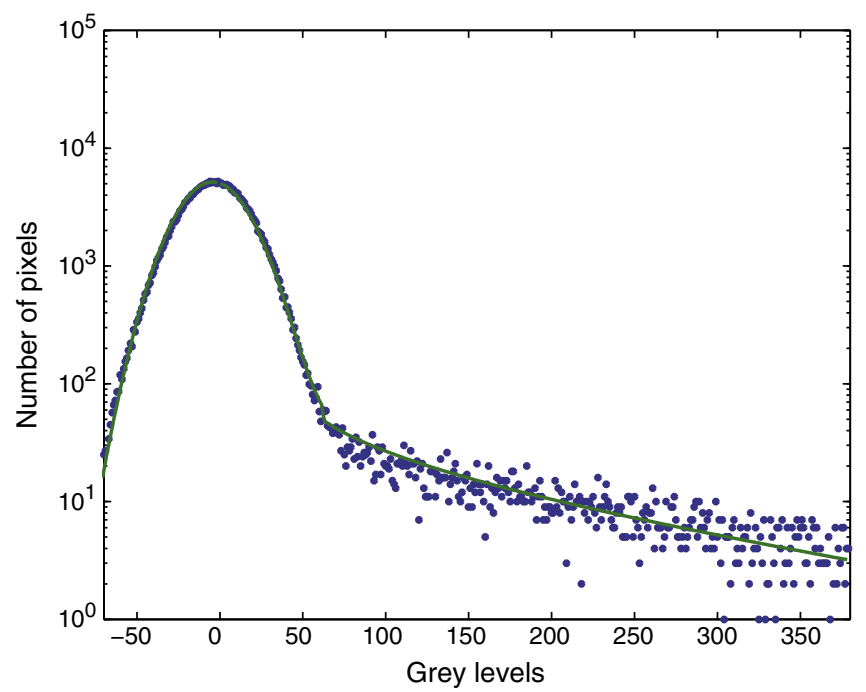

Fig. 5. Histogram of grey levels for a dark image of the Hamamatsu EMCCD (blue dots) and corresponding fit (green curve). 
We report in Fig. 6 the probabilities involved in the thresholding procedure of the Hamamatsu EMCCD camera, similarly to Fig. 3. Note that in Fig. 6a the chosen value for the mean illumination level on one image $(\mu=0.1 \mathrm{ph} / \mathrm{pix})$ is lower than the dark noise variance of the EMCCD; in that case the comparison with the analytical value given by Eq. (6) shows that this choice for $\mu$ is not the best since the result of the calculation gives 0.2 , which is $\neq 0.1$ ! Indeed it can easily be verified by iteration that $\mu=0.2 p h / p i x$ (thus just above the noise standard deviation), corresponds to the optimal value (case of Fig. 6b). With this value for the mean number of photons per pixel, we find from the intersection of $P(x \mid 0)(1-\mu)$ and $P(x \mid 1) \mu$ the optimal threshold value to be applied in single photon detection regime, that is $T=46$ grey levels.

For a comparison with the Andor EMCCD, we show in Fig. 7 the three false detection probabilities, the total probability of error on the pixel, plotted as a function of the mean number of photons per pixel $\mu$, for $T=46 g l$, together with the total relative error in detection (black curve), which in this case lies always above 0.39. In general, we notice that after the optimal thresholding procedure, the probability values of false detection are double with respect to those of Fig. 4. Here it turns out that for a recorded image illuminated with a mean number of 0.2 photons per pixel, we have a total false detection probability of about $7 \%$.

\section{Conclusions}

In this paper we have presented a clear and straightforward thresholding graphical method for photon counting with EMCCDs. The threshold level $T$ and consequently-thanks to an iterative optimization process-the best mean level of illumination $\mu$ to use are derived with a simple procedure based on the graphical visualization of the probabilities involved in the single photon counting regime in the detection errors on 1 pixel. Moreover by comparing the results with the analytical work presented in [9], we have shown that the obtained parameters $T$ and $\mu$ necessary for the thresholding procedure to be used in the single photon detection process, are indeed those that minimize the quadratic error for 1 pixel on the sum of the images.

We have applied this graphical method to two different EMCCDs, therefore comparing the noise statistics of the dark images recorded, and the false detection probabilities after applying the optimal graphical procedure. We could in this way notice how the demo EMCCD from Hamamatsu revealed to be less adequate with respect to the selected Andor camera for single photon counting measurements because of the larger readout noise, and the larger probabilities of errors that would occur in detection. In particular in cases where for instance the quantum properties of the twin beam spatial correlations have to be analyzed, the EMCCD with very low false detection probabilities would certainly be the best choice for the reach of the purpose. On the other hand note in an aside that thanks to their high dynamic range Hamamatsu EMCCDs are especially suitable for applications in low light luminescence imaging and high dynamic range brightfield imaging in life sciences, materials research and industrial imaging.

We conclude by saying that the graphical method for the derivation of the threshold proposed here can be applied more generally to any kind of EMCCD camera with similar types of sensor, but different electronics and thus noise characteristics. In general it should become a valuable tool for any user of EMCCD working with single photon light levels for any kind of applications.

\section{Acknowledgement}

O.J., J.-L B. and P.D.T acknowledge the financial support of the Future and Emerging Technologies (FET) programme within the Seventh Framework Programme for Research of the European Commission, under the FET-Open grant agreement HIDEAS, number FP7-ICT-221906.
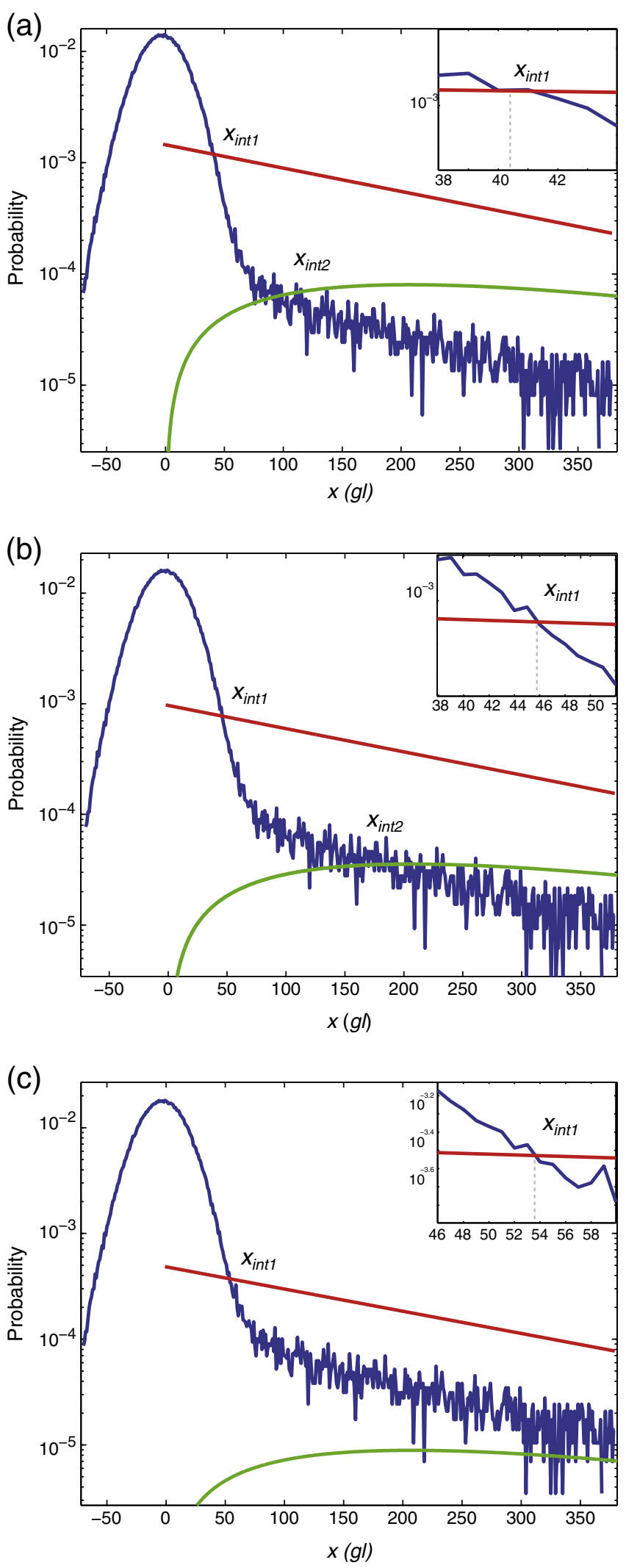

Fig. 6. Probabilities $\left(p(x \mid 0)(1-\mu)\right.$ in blue, $p(x \mid 1) \mu$ in red, and $p(x \mid 2) \mu^{2} / 2$ in green) involved in the thresholding procedure for the Hamamatsu EMCCD demo, for three different cases of illumination; (a) $\mu=0.3 \mathrm{ph} / \mathrm{pix}$, (b) $\mu=0.2 \mathrm{ph} / \mathrm{pix}$, (c) $\mu=0.1 \mathrm{ph} / \mathrm{pix}$. 


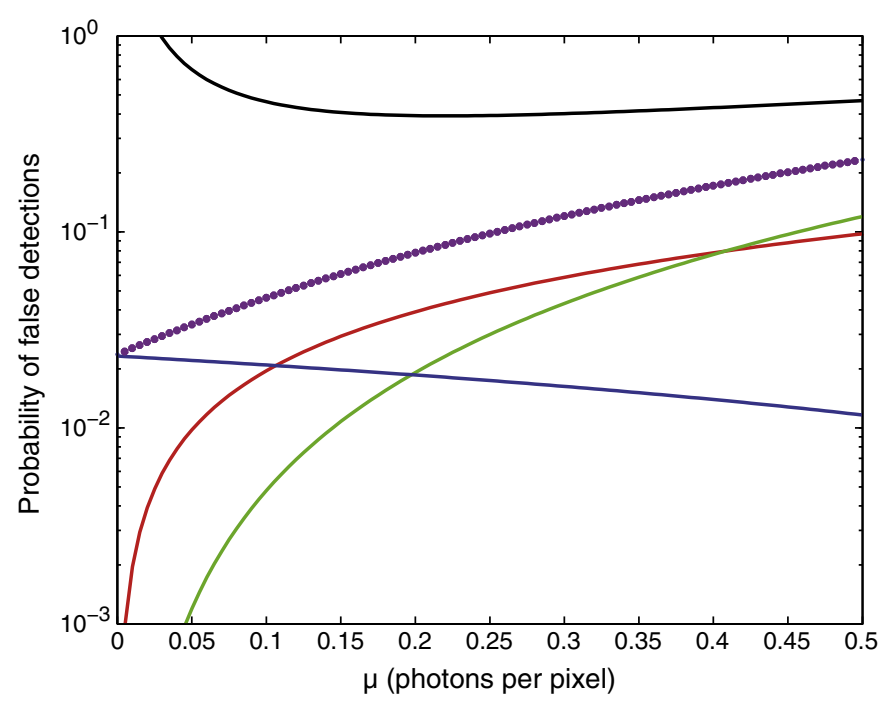

Fig. 7. False detection probabilities $P_{0}$ (blue curve), $P_{1}$ (red curve) and $P_{2}$ (green curve), and total probability of error (dotted curve), plotted as a function of $\mu$, for $T=46 \mathrm{gl}$ in the case of the Hamamatsu demo EMCCD. The black dotted curve represents the relative total error in detection.

\section{References}

[1] S.K. Madan, B. Bhaumik, J.M. Vasi, IEEE Transactions on Electron Devices ED-30 (1983) 694

[2] J.-L. Blanchet, F. Devaux, L. Furfaro, E. Lantz, Physical Review Letters (2008) 233604.

[3] J.-L. Blanchet, F. Devaux, L. Furfaro, E. Lantz, Physical Review A (2010) 043825.

[4] L. Zhang, L. Neves, J.S. Lundeen, I.A. Walmsey, Journal of Physics B: Atomic, Molecular and Optical Physics (2009) 114011.

[5] K. Matsuo, M.C. Teich, B.E.A. Saleh, IEEE Transactions on Electron Devices ED-32 (1985) 2615.

[6] J.N. Hollenhorst, IEEE Transactions on Electron Devices (1990) 781.

[7] J. Hynecek, T. Nishiwaki, IEEE Transactions on Electron Devices (2003) 239.

[8] A.G. Basden, C.A. Haniff, C.D. Mackay, Monthly Notices of the Royal Astronomical Society (2003) 985.

[9] E. Lantz, J.-L. Blanchet, L. Furfaro, F. Devaux, Monthly Notices of the Royal Astronomical Society (2008) 2262. 